\title{
A New Sequential Algorithm for Hyperspectral Endmember Extraction
}

\author{
Qian Du, Senior Member, IEEE
}

\begin{abstract}
Endmember extraction is an important step in spectral mixture analysis when endmembers are unknown. Endmembers are usually assumed to be pure pixels present in an image scene. Under this circumstance, endmember extraction is to find the most distinctive pixels. To make the searching process more efficient, the sequential forward search (SFS) method is generally used, where the next endmember is determined with a certain criterion based on the currently extracted endmember set. This letter proposes a new criterion which is related to the estimated endmember abundances. Compared to other sequential endmember extraction algorithms, the proposed method can find all the different endmembers faster. This letter also proposes to use the sequential forward floating search method as the substitute of SFS, which can improve the performance of all the sequential endmember extraction algorithms.
\end{abstract}

Index Terms-Endmember extraction, hyperspectral imagery, linear mixture analysis, sequential forward floating search (SFFS), sequential forward searching.

\section{INTRODUCTION}

$\mathbf{L}$ INEAR mixture analysis is a widely used technique in remote sensing image analysis. It is particularly useful for hyperspectral imagery due to the fact that the high data dimensionality relaxes the limitation imposed on the number of endmembers that can be resolved. Endmember signatures need to be known for linear unmixing. In many practical situations where these signatures are unknown, endmembers have to be estimated directly from the image scene under study in an unsupervised fashion.

Many endmember extraction algorithms find distinctive image pixels as endmember signatures [1]. They usually implicitly assume that pure pixels are present in an image scene. These algorithms may use different criteria to measure pixel distinctiveness. Two major criteria are multidimensional geometrybased simplex volume maximization and pixel spectral signature similarity. The well-known N-FINDR algorithm employs the first criterion, and it is based on the fact that, in a $p$-dimensional space, the $p$-volume simplex formed by the purest pixels has the largest volume [2]. In the second criterion, a certain pixel spectral similarity metric is defined. For instance, the automatic target generation process (ATGP) [3] searches the most distinctive pixels by using the orthogonal subspace projection (OSP) approach, and the pixel with the

Manuscript received October 20, 2011; accepted November 30, 2011. Date of publication January 23, 2012; date of current version May 7, 2012.

The author is with the Geosystems Research Institute, High Performance Computing Collaboratory, and the Department of Electrical and Computer Engineering, Mississippi State University, Mississippi State, MS 39762 USA (e-mail: du@ece.msstate.edu).

Color versions of one or more of the figures in this paper are available online at http://ieeexplore.ieee.org.

Digital Object Identifier 10.1109/LGRS.2011.2178815 largest projection in the orthogonal subspace of a set of pixels is selected as endmember. Vertex component analysis (VCA) is similar to ATGP by adopting the idea of OSP maximization but with dimensionality reduction [4]. The fully constrained least squares linear unmixing (FCLSLU) finds an endmember by determining the pixel with the largest reconstruction error [5]. Similarly, the iterative error analysis (IEA) [6] is an endmember extraction method based on spectral signature similarity. The algorithms for endmember extraction may also differ in the implementation mode, i.e., parallel or sequential. In the parallel mode, endmembers are determined simultaneously, while in the sequential mode, endmembers are determined one after another. The original N-FINDR algorithm is implemented in the parallel mode, and its sequential version is the simplex growing algorithm (SGA) [7]. The relationship between these two criteria, simplex volume maximization and spectral signature similarity, has been analyzed in [8]. They are theoretically equivalent. The relationship between ATGP, FCLSLU, and VCA was analyzed in [9]. The performance discrepancy from parallel and sequential modes and the use of dimensionality reduction were also investigated in [9].

Note that there is another group of algorithms without pure pixel assumption, finding endmembers that may not be image pixels. The minimum-volume transform (MVT) in [10] belongs to this type, which is to search for nonpixel endmembers that construct a simplex enclosing the maximum data points with the minimum volume. Several MVT-based algorithms are reported, such as minimum-volume-constrained nonnegative matrix factorization [11] and minimum-volume simplex analysis [12].

In this letter, the author limits the discussion on the algorithms finding image pixels as endmembers sequentially. To find $k$ endmember pixels from $N$ pixels is a traditional feature selection problem. Since exhaustive search is not feasible to high-dimensional problems, research has been focused on suboptimal search methods. Two conventional approaches are the "bottom-up" sequential forward search (SFS) and the "topdown" sequential backward search (SBS). When the number of endmembers to be extracted is unknown, SBS is not applicable. Therefore, the SFS is widely used for the sequential endmember extraction algorithms: It starts with the first endmember and then adds an additional endmember in each iteration based on certain criteria; an algorithm is usually initialized by the first endmember, e.g., the pixel with the largest norm.

In this letter, the author proposes a new sequential endmember extraction algorithm based on a criterion related to endmember abundances. In addition, the author proposes to use the sequential forward floating search (SFFS) method for distinctive pixel searching. The proposed algorithm is compared with two well-known sequential algorithms: VCA (using pixel similarity criterion) and SGA (using simplex volume criterion). 


\section{PROpOSED APPROACH}

\section{A. Proposed Endmember Extraction Criterion}

Assume that there are $p$ pure materials present in an image scene. Based on the linear mixture model, a pixel $\mathrm{x}$ can be considered as the mixing result of the endmembers of the $p$ materials. Let the endmember matrix be $\mathbf{S}=\left[\mathbf{s}_{1}, \mathbf{s}_{2}, \ldots, \mathbf{s}_{p}\right]$. The pixel $\mathrm{x}$ can be expressed as

$$
\mathbf{x}=\mathbf{S} \boldsymbol{\alpha}+\mathbf{n}
$$

where $\boldsymbol{\alpha}=\left(\alpha_{1} \alpha_{2} \cdots \alpha_{p}\right)^{\mathrm{T}}$ is the abundance vector and $\mathbf{n}$ is the uncorrelated white noise with $E(\mathbf{n})=\mathbf{0}$ and $\operatorname{Cov}(\mathbf{n})=$ $\sigma^{2} \mathbf{I}$ (I is an identity matrix). The least squares estimation of $\boldsymbol{\alpha}$, denoted as $\hat{\boldsymbol{\alpha}}$, can be obtained as

$$
\hat{\boldsymbol{\alpha}}=\left(\mathbf{S}^{\mathrm{T}} \mathbf{S}\right)^{-1} \mathbf{S}^{\mathrm{T}} \mathbf{x} .
$$

According to Johnson and Wiechern [13], the stochastic features of $\boldsymbol{\alpha}$ include

$$
E(\hat{\boldsymbol{\alpha}})=\boldsymbol{\alpha} \quad \operatorname{Cov}(\hat{\boldsymbol{\alpha}})=\sigma^{2}\left(\mathbf{S}^{\mathrm{T}} \mathbf{S}\right)^{-1} .
$$

If there are $q$ materials present and $q>p$, it means that only $p$ signatures are known. Then, if the same linear mixture equation (1) is used, noise $\mathbf{n}$ cannot be white noise anymore because $\mathbf{n}$ is the reconstruction residual including signal components. Instead, $\operatorname{Cov}(\mathbf{n})=\sigma^{2} \boldsymbol{\Sigma}$, where $\boldsymbol{\Sigma}$ is the noise covariance matrix. In this case, the abundances of the $p$ signatures can be estimated using the weighted least squares solution as

$$
\hat{\boldsymbol{\alpha}}=\left(\mathbf{S}^{\mathrm{T}} \boldsymbol{\Sigma}^{-1} \mathbf{S}\right)^{-1} \mathbf{S}^{\mathrm{T}} \boldsymbol{\Sigma}^{-1} \mathbf{x} .
$$

According to [13], the first- and second-order moments of $\hat{\boldsymbol{\alpha}}$ are

$$
E(\hat{\boldsymbol{\alpha}})=\boldsymbol{\alpha} \quad \operatorname{Cov}(\hat{\boldsymbol{\alpha}})=\sigma^{2}\left(\mathbf{S}^{\mathrm{T}} \boldsymbol{\Sigma}^{-1} \mathbf{S}\right)^{-1} .
$$

If signals are assumed to be deterministic, then $\Sigma$ can be replaced with the data covariance matrix.

The estimated abundance statistics can be used for endmember extraction, in which only the second case $(q>p)$ is applicable. Intuitively, the selected pixel endmembers should let the deviation of $\hat{\boldsymbol{\alpha}}$ from the actual $\boldsymbol{\alpha}$ as small as possible, which is equivalent to minimizing the trace of the covariance, i.e.,

$$
\underset{\mathbf{S}}{\arg \min }\left\{\operatorname{trace}\left[\left(\mathbf{S}^{\mathrm{T}} \boldsymbol{\Sigma}^{-1} \mathbf{S}\right)^{-1}\right]\right\}
$$

based on (5). The resulting endmember extraction algorithm is referred to as the minimum estimated abundance covariance (MEAC) method.

The basic steps in the MEAC method can be described as follows.

1) Perform dimensionality reduction with maximum-noisefraction transform as needed.

2) To find the first endmember $\mathbf{s}_{1}$, let the current endmember set be $\mathbf{S}_{1}=[\mathbf{x}]$ temporarily to test each pixel $\mathbf{x}$; if $\mathbf{S}_{1}=$ $\left[\mathbf{x}_{\min }^{(1)}\right]$ is the one yielding the minimum value for (6), then $\mathbf{s}_{1}=\mathbf{x}_{\min }^{(1)}$.

3) To find the second endmember $\mathbf{s}_{2}$, let $\mathbf{S}_{2}=\left[\mathbf{S}_{1} \mathbf{x}\right]$ temporarily to test each pixel $\mathbf{x}$; if $\mathbf{S}_{2}=\left[\mathbf{S}_{1} \mathbf{x}_{\text {min }}^{(2)}\right]$ is the one yielding the minimum value for (6), then $\mathbf{s}_{2}=\mathbf{x}_{\min }^{(2)}$.

4) Continue on step 3) until the number of endmembers in $S$ is sufficient.

Note that the MEAC algorithm does not need to identify an initial condition. It can be automatically initiated.

\section{B. Fast Processing}

Assume that $i$ endmembers have been selected, which construct a signature matrix $\mathbf{S}_{i}$. To search for the $(i+1)$ th endmember, each pixel has to be tested, and a matrix inversion computation is needed in (6). To save computational cost, $\left(\mathbf{S}_{i+1}^{\mathrm{T}} \boldsymbol{\Sigma}^{-1} \mathbf{S}_{i+1}\right)^{-1}$ can be calculated based on $\left(\mathbf{S}_{i}^{\mathrm{T}} \boldsymbol{\Sigma}^{-1} \mathbf{S}_{i}\right)^{-1}$, where $\mathbf{S}_{i+1}=\left[\mathbf{S}_{i} \mathbf{S}_{i+1}\right]$. For convenience, define $\mathbf{R}_{i}=\mathbf{S}_{i}^{\mathrm{T}} \boldsymbol{\Sigma}^{-1} \mathbf{S}_{i}$ and $\mathbf{R}_{i+1}=\mathbf{S}_{i+1}^{\mathrm{T}} \boldsymbol{\Sigma}^{-1} \mathbf{S}_{i+1}$. They can be related as

$$
\mathbf{R}_{i+1}=\left[\begin{array}{cc}
\mathbf{R}_{i} & \mathbf{r}_{i, i+1} \\
\mathbf{r}_{i, i+1}^{\mathrm{T}} & r_{i+1, i+1}
\end{array}\right]
$$

where $r_{i+1, i+1}=\mathbf{s}_{i+1}^{\mathrm{T}} \boldsymbol{\Sigma}^{-1} \mathbf{s}_{i+1}$ and $\mathbf{r}_{i, i+1}=\mathbf{S}_{i}^{\mathrm{T}} \boldsymbol{\Sigma}^{-1} \mathbf{s}_{i+1}$. Using the following partitioned matrix inversion formula, $\mathbf{R}_{i+1}^{-1}$ can be calculated by modifying $\mathbf{R}_{i}^{-1}$ with $r_{i+1, i+1}$ and $\mathbf{r}_{i, i+1}$.

Let a matrix $\mathbf{A}$ be partitioned as $\mathbf{A}=\left[\begin{array}{ll}\mathbf{A}_{11} & \mathbf{A}_{12} \\ \mathbf{A}_{21} & \mathbf{A}_{22}\end{array}\right]$. Then, its inverse matrix $\mathbf{A}^{-1}$ can be calculated as (8), shown at the bottom of the page. Let $\mathbf{A}_{11}=\mathbf{R}_{i}, \mathbf{A}_{22}=r_{i+1, i+1}$, $\mathbf{A}_{12}=\mathbf{r}_{i, i+1}$, and $\mathbf{A}_{21}=\mathbf{r}_{i, i+1}^{\mathrm{T}}$. According to (8), we have (9), shown at the bottom of the page. In (9),

$$
\mathbf{A}^{-1}=\left[\begin{array}{cc}
\left(\mathbf{A}_{11}-\mathbf{A}_{12} \mathbf{A}_{22}^{-1} \mathbf{A}_{21}\right)^{-1} & -\left(\mathbf{A}_{11}-\mathbf{A}_{12} \mathbf{A}_{22}^{-1} \mathbf{A}_{21}\right)^{-1} \mathbf{A}_{12} \mathbf{A}_{22}^{-1} \\
-\left(\mathbf{A}_{22}-\mathbf{A}_{21} \mathbf{A}_{11}^{-1} \mathbf{A}_{12}\right)^{-1} \mathbf{A}_{21} \mathbf{A}_{11}^{-1} & \left(\mathbf{A}_{22}-\mathbf{A}_{21} \mathbf{A}_{11}^{-1} \mathbf{A}_{12}\right)^{-1}
\end{array}\right]
$$

$$
\mathbf{R}_{i+1}^{-1}=\left[\begin{array}{cc}
\left(\mathbf{R}_{i}-\mathbf{r}_{i, i+1} r_{i+1, i+1} \mathbf{r}_{i, i+1}^{\mathrm{T}}\right)^{-1} & -\left(\mathbf{R}_{i}-\mathbf{r}_{i, i+1} r_{i+1, i+1} \mathbf{r}_{i, i+1}^{\mathrm{T}}\right)^{-1} \mathbf{r}_{i, i+1} r_{i+1, i+1}^{-1} \\
-\left(r_{i+1, i+1}-\mathbf{r}_{i, i+1}^{\mathrm{T}} \mathbf{R}_{i}^{-1} \mathbf{r}_{i, i+1}\right)^{-1} \mathbf{r}_{i, i+1}^{\mathrm{T}} \mathbf{R}_{i}^{-1} & \left(r_{i+1, i+1}-\mathbf{r}_{i, i+1}^{\mathrm{T}} \mathbf{R}_{i}^{-1} \mathbf{r}_{i, i+1}\right)^{-1}
\end{array}\right]
$$


$\alpha=\left(r_{i+1, i+1}-\mathbf{r}_{i, i+1}^{\mathrm{T}} \mathbf{R}_{i}^{-1} \mathbf{r}_{i, i+1}\right)^{-1}$ is a scalar. Using Woodbury's formula

$$
\begin{aligned}
\left(\mathbf{R}_{i}\right. & \left.-\mathbf{r}_{i, i+1} r_{i+1, i+1} \mathbf{r}_{i, i+1}^{\mathrm{T}}\right)^{-1} \\
& =\mathbf{R}_{i}^{-1}+\mathbf{R}_{i}^{-1} \mathbf{r}_{i, i+1}\left(r_{i+1, i+1}^{-1}-\mathbf{r}_{i, i+1}^{\mathrm{T}} \mathbf{R}_{i}^{-1} \mathbf{r}_{i, i+1}\right)^{-1} \mathbf{r}_{i, i+1}^{\mathrm{T}} \mathbf{R}_{i}^{-1} \\
& =\mathbf{R}_{i}^{-1}+\alpha \mathbf{R}_{i}^{-1} \mathbf{r}_{i, i+1} \mathbf{r}_{i, i+1}^{\mathrm{T}} \mathbf{R}_{i}^{-1}
\end{aligned}
$$

and (9) becomes

$$
\mathbf{R}_{i+1}^{-1}=\left[\begin{array}{cc}
\mathbf{R}_{i}^{-1}+\alpha \mathbf{R}_{i}^{-1} \mathbf{r}_{i, i+1} \mathbf{r}_{i, i+1}^{\mathrm{T}} \mathbf{R}_{i}^{-1} & -\alpha \mathbf{R}_{i}^{-1} \mathbf{r}_{i, i+1} \\
-\alpha \mathbf{r}_{i, i+1}^{\mathrm{T}} \mathbf{R}_{i}^{-1} & \alpha
\end{array}\right] .
$$

Thus, all the elements in (10) can be generated by simple matrix multiplication. In this case, no operation of matrix inversion is needed when reaching the final $\mathbf{R}_{i+1}^{-1}$. Based on (11)

$$
\begin{aligned}
\operatorname{trace}\left(\mathbf{R}_{i+1}^{-1}\right)= & \operatorname{trace}\left(\mathbf{R}_{i}^{-1}+\alpha \mathbf{R}_{i}^{-1} \mathbf{r}_{i, i+1} \mathbf{r}_{i, i+1}^{\mathrm{T}} \mathbf{R}_{i}^{-1}\right)+\alpha \\
= & \operatorname{trace}\left(\mathbf{R}_{i}^{-1}\right) \\
& +\alpha \cdot \operatorname{trace}\left(\mathbf{R}_{i}^{-1} \mathbf{r}_{i, i+1} \mathbf{r}_{i, i+1}^{\mathrm{T}} \mathbf{R}_{i}^{-1}\right)+\alpha \\
= & \operatorname{trace}\left(\mathbf{R}_{i}^{-1}\right)+\alpha \mathbf{r}_{i, i+1}^{\mathrm{T}} \mathbf{R}_{i}^{-1} \mathbf{R}_{i}^{-1} \mathbf{r}_{i, i+1}+\alpha
\end{aligned}
$$

with $\mathbf{R}_{1}^{-1}=\left(\mathbf{S}_{1} \boldsymbol{\Sigma}^{-1} \mathbf{S}_{1}^{\mathrm{T}}\right)^{-1}=\left(\mathbf{s}_{1} \boldsymbol{\Sigma}^{-1} \mathbf{s}_{1}^{\mathrm{T}}\right)^{-1}$ being a scalar operation. This means that the MEAC algorithm does not need additional matrix inversion operation, and the trace value in the current step can be easily updated using the one in the previous step.

\section{Alternative Searching Strategy}

The SFS-based endmember extraction algorithms suffer from the so-called "nesting effect" and are sensitive to the initial condition. In other words, any inaccuracy in selected endmembers will be carried on or even magnified when more endmembers are extracted. Attempts to prevent the nesting of feature subsets led to the development of SFFS algorithm [14]: After a step of SFS generating $k$ selected features, SBS is applied to ensure that the $(k-1)$ selected features for finding the $k$ th feature are the optimal. It can be generalized to the plus-l-minus-r search [15].

As in the existing sequential endmember extraction algorithms, such as VCA and SGA, and the proposed MEAC algorithm, the basic steps of the SFS for endmember extraction can be summarized as follows.

1) If needed, initialize the algorithm with a randomly selected pixel or the one with the maximum norm as the first endmember.

2) Find the next pixel that is the most dissimilar to the current selected endmember(s) based on a certain criterion.

3) Stop if the number of selected endmembers is $k_{\max }$.

The block diagram of the SFFS algorithm is shown in Fig. 1. The basic steps of the SFFS algorithm for endmember extraction can be described as follows.

1) Initialize the algorithm as needed.

2) Forward step: If the current endmember set includes $k$ endmembers, find the $(k+1)$ th endmember using the SFS algorithm.

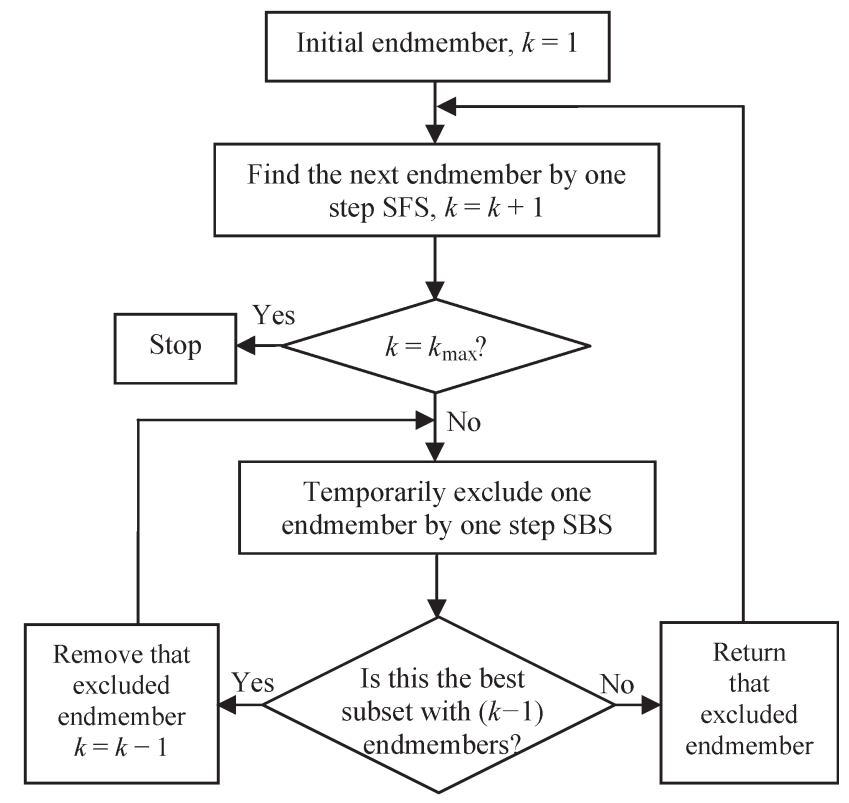

Fig. 1. Diagram of the SFFS algorithm.

3) Backward step: If the current endmember set includes $(k+1)$ endmembers, conditionally delete one endmember to see if the remaining $k$-endmember set is historically the best. If yes, leave this endmember out, let $k=k-1$, and continue such SBS step. If no, return this endmember back, and go to the forward step.

4) Stop if the selected endmembers are enough.

The criterion for the "best" set depends on the one used to gauge pixel distinctiveness. For VCA, it is the maximum norm in the endmember orthogonal subspace. For SGA, it is the maximum simplex volume. For MEAC, it is the minimum trace of estimated covariance matrix. The advantage of the SFS is its computational efficiency. Although the SFFS may result in a better feature set, its computational cost is significantly increased due to the included SBS steps.

\section{EXPERIMENTS}

In the experiments, the author chose two popular sequential algorithms, VCA and SGA, for comparison purposes. This is because of the following facts: 1) they employ two different criteria to measure pixel distinctiveness and 2) they have good performance in general.

Based on our experience, as long as the number of endmembers to be searched $k$ is sufficiently large (which is also equal to the number of iterations in SFS), most of existing endmember extraction algorithms can find all the endmember types that exist in an image scene. However, it is important to find these different endmember types in the very early stages or as early as possible. An algorithm that can find distinctive pixels sooner is more capable of finding pixels with subtle difference. Moreover, the number of endmembers $k_{\max }$ is unknown in practice, and we can use an estimated virtual dimensionality (VD) as a reference [16], [17] (e.g., $k_{\max }=1-2$ VDs). All the endmembers must be found before the algorithm is manually terminated with such an estimated $k_{\text {max }}$. Obviously, endmember extraction performance should also be assessed by comparing with the actual endmember signatures if available. 


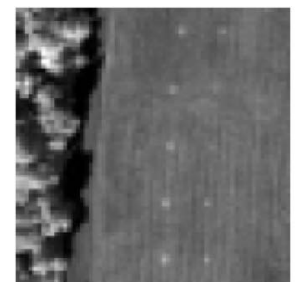

(a)

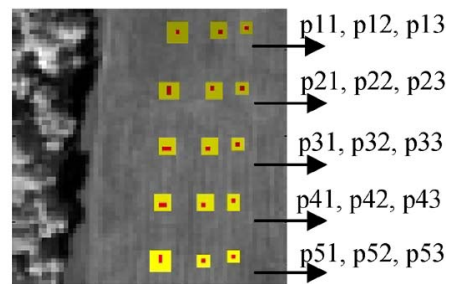

(b)
Fig. 2. HYDICE image scene with 15 panels. (a) Original image scene. (b) Panel locations.

\section{A. HYDICE Experiment}

The Hyperspectral Digital Imagery Collection Experiment (HYDICE) subimage scene of size $64 \times 64$ shown in Fig. 2(a) has about $1.5-\mathrm{m}$ spatial resolution. The spectral coverage is 0.4-2.5 $\mu \mathrm{m}$. After the water absorption and low-SNR bands were removed, 169 bands were used. This scene includes 15 panels arranged in a $5 \times 3$ matrix. The three panels in the same row $p_{i a}, p_{i b}, p_{i c}$ were made from the same material of sizes $3 \mathrm{~m} \times 3 \mathrm{~m}, 2 \mathrm{~m} \times 2 \mathrm{~m}$, and $1 \mathrm{~m} \times 1 \mathrm{~m}$, respectively, which can be considered as one class $P_{i}$ for $1 \leq i \leq 5$. The pixel-level ground truth map in Fig. 2(b) shows the precise locations of pure panel pixels.

In addition to the five panel endmembers, tree, and grassland, there is an anomaly in trees. In this experiment, the author mainly focuses on how fast an endmember extraction algorithm can find all the panel endmembers because all the endmember types can be accurately found if MEAC, VCA, or SGA is continuously executed. As shown in Fig. 3(a), MEAC could find all the panel endmembers (in the leftmost column) after six iterations, and the first endmember found in the first iteration was an anomaly in the tree line; in addition, after ten iterations, other endmembers such as tree and grassland were found. Applying SFFS for MEAC did not bring about further improvement. In Fig. 3(b), VCA could find all the panel endmembers after nine iterations; when using SFFS, eight iterations were needed. For SGA in Fig. 3(c), 20 iterations were needed if SFS was the searching method; when SFFS was used, only 15 iterations were required to determine all the panel endmembers. Note that the VD estimate for this image scene was around ten. If MEAC or VCA was chosen for endmember extraction, there was no problem to find all the endmembers if the algorithm was terminated after ten iterations.

\section{B. Cuprite Experiment}

The AVIRIS Cuprite subimage scene of size $350 \times 350$ is shown in Fig. 4. The spatial resolution is $20 \mathrm{~m}$. Originally, it has 224 bands with 0.4 - to $2.5-\mu \mathrm{m}$ spectral range. After the water absorption and low-SNR bands were removed, 189 bands were used. This image scene is mineralogically well understood [18]. The spectral library is available for this image scene. The VD estimate was about 22, so 30 endmembers were extracted. After comparing with the signatures in the library, 19 endmembers were matched as listed in Table I.

To compare the accuracies of the extracted endmembers, the spectral angle between an extracted endmember and the corresponding signature in the library was computed, and the spectral angles of all the 19 endmembers were averaged.

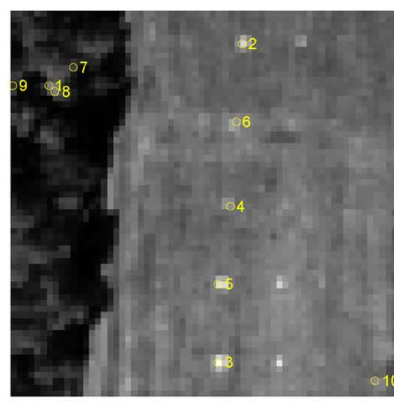

MEAC (SFS)

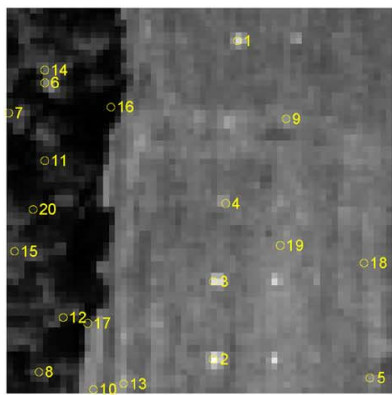

VCA (SFS)

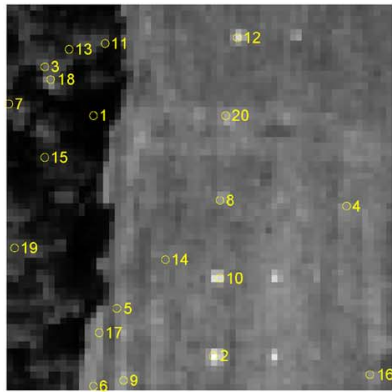

SGA (SFS)

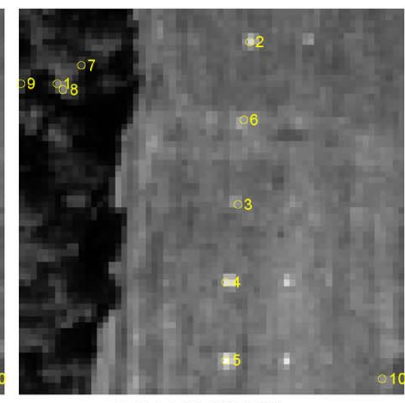

MEAC (SFFS)

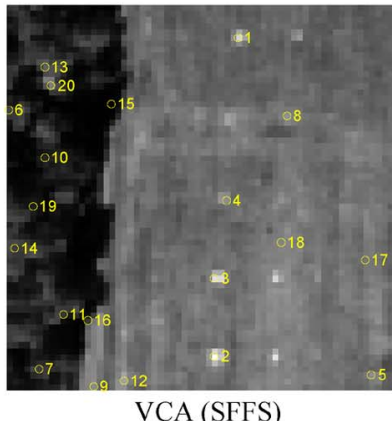

(b)

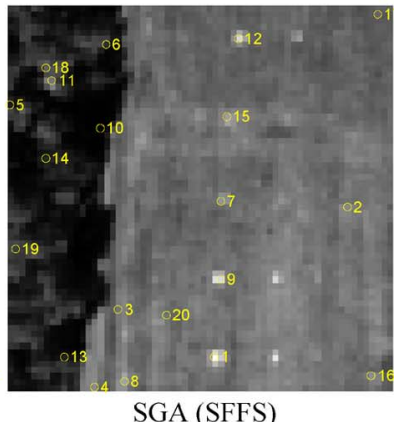

(c)

Fig. 3. HYDICE extracted endmember locations and orders.

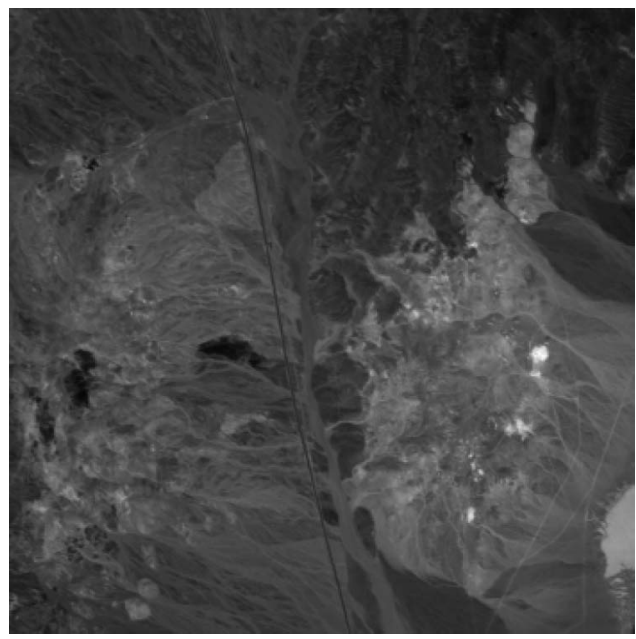

Fig. 4. Cuprite image scene used in the experiment.

Table II lists the results from MEAC, VCA, and SGA using SFS and SFFS. Obviously, the extracted endmember signatures were closer to those in the library using SFFS. Among the three sequential endmember extraction algorithms, they 
TABLE I

INDICES OF MATCHED ENDMEMBERS

\begin{tabular}{|c|c|c|c|}
\hline Index & Endmember & Index & Endmember \\
\hline 1 & alunite3 & 11 & jarosit8 \\
\hline 2 & alunite4 & 12 & montmor4 \\
\hline 3 & budding1 & 13 & nontronite2 \\
\hline 4 & calcite3 & 14 & pyrophy2 \\
\hline 5 & kaolini1 & 15 & chalcedony \\
\hline 6 & kaolini2 & 16 & dickite2 \\
\hline 7 & muscovi1 & 17 & andradi4 \\
\hline 8 & muscovi2 & 18 & dumortie \\
\hline 9 & muscovi3 & 19 & sphene \\
\hline 10 & chlorite6 & & \\
\hline
\end{tabular}

TABLE II

Spectral Angles Between the Extracted ENDMEMbers AND the Signatures in the U.S. GeOlogical SuRVey SPeCtral LibraRy

\begin{tabular}{|c|c|c|c|c|c|c|}
\hline & $\begin{array}{c}\text { MEAC } \\
(\text { SFS })\end{array}$ & $\begin{array}{c}\text { MEAC } \\
(\text { SFFS })\end{array}$ & $\begin{array}{c}\text { VCA } \\
(\text { SFS })\end{array}$ & $\begin{array}{c}\text { VCA } \\
(\text { SFFS })\end{array}$ & $\begin{array}{c}\text { SGA } \\
(\text { SFS })\end{array}$ & $\begin{array}{c}\text { SGA } \\
(\text { SFFS })\end{array}$ \\
\hline 1 & $3.64^{\circ}$ & $3.64^{\circ}$ & $3.64^{\circ}$ & $3.64^{\circ}$ & $3.64^{\circ}$ & $3.64^{\circ}$ \\
\hline 2 & $6.55^{\circ}$ & $6.55^{\circ}$ & $6.55^{\circ}$ & $6.03^{\circ}$ & $6.55^{\circ}$ & $6.55^{\circ}$ \\
\hline 3 & $4.09^{\circ}$ & $4.09^{\circ}$ & $4.16^{\circ}$ & $4.11^{\circ}$ & $4.09^{\circ}$ & $4.09^{\circ}$ \\
\hline 4 & $5.09^{\circ}$ & $5.09^{\circ}$ & $5.21^{\circ}$ & $5.17^{\circ}$ & $5.09^{\circ}$ & $5.09^{\circ}$ \\
\hline 5 & $7.73^{\circ}$ & $7.73^{\circ}$ & $7.73^{\circ}$ & $7.73^{\circ}$ & $7.73^{\circ}$ & $7.73^{\circ}$ \\
\hline 6 & $6.75^{\circ}$ & $6.75^{\circ}$ & $6.75^{\circ}$ & $6.75^{\circ}$ & $6.75^{\circ}$ & $6.12^{\circ}$ \\
\hline 7 & $5.40^{\circ}$ & $4.67^{\circ}$ & $4.67^{\circ}$ & $5.31^{\circ}$ & $5.60^{\circ}$ & $5.60^{\circ}$ \\
\hline 8 & $3.91^{\circ}$ & $3.91^{\circ}$ & $3.88^{\circ}$ & $3.78^{\circ}$ & $3.91^{\circ}$ & $3.91^{\circ}$ \\
\hline 9 & $6.94^{\circ}$ & $6.67^{\circ}$ & $6.67^{\circ}$ & $6.67^{\circ}$ & $6.98^{\circ}$ & $6.98^{\circ}$ \\
\hline 10 & $7.66^{\circ}$ & $7.22^{\circ}$ & $7.60^{\circ}$ & $7.22^{\circ}$ & $7.88^{\circ}$ & $7.88^{\circ}$ \\
\hline 11 & $4.63^{\circ}$ & $4.78^{\circ}$ & $4.78^{\circ}$ & $4.78^{\circ}$ & $5.76^{\circ}$ & $6.00^{\circ}$ \\
\hline 12 & $3.98^{\circ}$ & $3.98^{\circ}$ & $5.47^{\circ}$ & $5.47^{\circ}$ & $5.31^{\circ}$ & $4.35^{\circ}$ \\
\hline 13 & $4.20^{\circ}$ & $4.07^{\circ}$ & $4.07^{\circ}$ & $3.95^{\circ}$ & $4.05^{\circ}$ & $4.05^{\circ}$ \\
\hline 14 & $6.32^{\circ}$ & $6.32^{\circ}$ & $6.16^{\circ}$ & $6.16^{\circ}$ & $4.60^{\circ}$ & $4.37^{\circ}$ \\
\hline 15 & $3.35^{\circ}$ & $3.41^{\circ}$ & $3.29^{\circ}$ & $2.41^{\circ}$ & $3.35^{\circ}$ & $3.35^{\circ}$ \\
\hline 16 & $6.27^{\circ}$ & $6.27^{\circ}$ & $6.30^{\circ}$ & $5.53^{\circ}$ & $6.27^{\circ}$ & $6.27^{\circ}$ \\
\hline 17 & $3.21^{\circ}$ & $3.21^{\circ}$ & $3.43^{\circ}$ & $3.43^{\circ}$ & $3.43^{\circ}$ & $3.43^{\circ}$ \\
\hline 18 & $4.53^{\circ}$ & $4.53^{\circ}$ & $5.11^{\circ}$ & $5.65^{\circ}$ & $4.65^{\circ}$ & $4.26^{\circ}$ \\
\hline 19 & $6.07^{\circ}$ & $4.33^{\circ}$ & $4.63^{\circ}$ & $3.18^{\circ}$ & $5.35^{\circ}$ & $5.35^{\circ}$ \\
\hline Ave. & $\mathbf{5 . 2 8}^{\circ}$ & $\mathbf{5 . 1 2}^{\circ}$ & $\mathbf{5 . 2 7}^{\circ}$ & $\mathbf{5 . 1 0}^{\circ}$ & $\mathbf{5 . 3 2}^{\circ}$ & $\mathbf{5 . 2 1}^{\circ}$ \\
\hline
\end{tabular}

basically provided similar accuracies when the same searching strategy was adopted, although the accuracy yielded by SGA was slightly lower than those by the other two methods.

\section{Conclusion}

In this letter, the author has proposed a new sequential endmember extraction algorithm based on the criterion of MEAC. It has the following advantages.

1) Its implementation is very easy because it can automatically determine the initial condition of the algorithm. Most sequential algorithms, such as VCA and SGA, require the initial condition as manual input; when the initial condition is changed, the algorithm output is also changed.

2) It is able to find different endmember types earlier. This means that the algorithm may be more adequately terminated if a VD estimate is used as the guidance for the number of endmembers to be extracted.

The accuracy of MEAC-extracted endmembers is similar to that of other widely used sequential algorithms, such as VCA and SGA. After using the fast processing strategy in
Section II-B, the computational cost of MEAC can be greatly reduced. Note that the same strategy in Section II-B can be applied for VCA and SGA such that their criterion function can be more simply evaluated based on the one in the previous stage.

The author has also proposed to use the SFFS method for sequential endmember extraction for MEAC, which is also applicable to VCA and SGA. SFFS can improve the accuracy of the extracted endmembers and may find different endmember types as the first several endmembers. However, it is computationally more expensive. Assume that $k$ endmembers have been extracted using SFS; if we want only $k-1$ endmembers, then the first $k-1$ out of $k$ endmembers will be the result. If the $k$ endmembers are determined by SFFS, there is no such easy correspondence to find $k-1$ endmembers; the algorithm basically has to be re-executed.

\section{REFERENCES}

[1] A. Plaza, P. Martinex, R. Perez, and J. Plaza, "A quantitative and comparative analysis of endmember extraction algorithms from hyperspectral data," IEEE Trans. Geosci. Remote Sens., vol. 42, no. 3, pp. 650-663, Mar. 2004.

[2] M. E. Winter, "N-finder: An algorithm for fast autonomous spectral endmember determination in hyperspectral data," in Proc. SPIE, 1999, vol. 3753, pp. 266-277.

[3] H. Ren and C.-I. Chang, "Automatic spectral target recognition in hyperspectral imagery," IEEE Trans. Aerosp. Electron. Syst., vol. 39, no. 4, pp. 1232-1249, Oct. 2003.

[4] J. M. P. Nascimento and J. M. Bioucas Dias, "Vertex component analysis: A fast algorithm to unmix hyperspectral data," IEEE Trans. Geosci. Remote Sens., vol. 43, no. 4, pp. 898-910, Apr. 2005.

[5] D. Heinz and C.-I. Chang, "Fully constrained least squares linear mixture analysis for material quantification in hyperspectral imagery," IEEE Trans. Geosci. Remote Sens., vol. 39, no. 3, pp. 529-545, Mar. 2001.

[6] R. A. Neville, K. Staenz, T. Szeredi, J. Lefebvre, and P. Hauff, "Automatic endmember extraction from hyperspectral data for mineral exploration," in Proc. 4th Int. Airborne Remote Sens. Conf. Exhib./21st Can. Symp. Remote Sens., Ottawa, ON, Canada, 1999, pp. 21-24.

[7] C.-I. Chang, C.-C. Wu, W.-M. Liu, and Y.-C. Ouyang, "A new growing method for simplex-based endmember extraction algorithm," IEEE Trans. Geosci. Remote Sens., vol. 44, no. 10, pp. 2804-2819, Oct. 2006.

[8] X. Tao, B. Wang, L. Zhang, and J. Zhang, "Orthogonal bases approach for the decomposition of mixed pixels in hyperspectral imagery," IEEE Geosci. Remote Sens. Lett., vol. 6, no. 2, pp. 219-223, Apr. 2009.

[9] Q. Du, N. Raksuntorn, N. H. Younan, and R. L. King, "Endmember extraction for hyperspectral image analysis," Appl. Opt., vol. 47, no. 28, pp. F77-F84, Oct. 2008.

[10] M. Craig, "Minimum-volume transforms for remotely sensed data," IEEE Trans. Geosci. Remote Sens., vol. 32, no. 3, pp. 542-552, May 1994.

[11] L. Miao and H. Qi, "Endmember extraction from highly mixed data using minimum volume constrained nonnegative matrix factorization," IEEE Trans. Geosci. Remote Sens., vol. 45, no. 3, pp. 765-777, Mar. 2007.

[12] J. Li and J. M. Bioucas Dias, "Minimum volume simplex analysis: A fast algorithm to unmix hyperspectral data," in Proc. IEEE Geosci. Remote Sens. Symp., Jul. 2008, vol. 3, pp. 250-253.

[13] R. A. Johnson and D. W. Wiechern, Applied Multivariate Statistical Analysis, 6th ed. Englewood Cliffs, NJ: Prentice-Hall, 2007.

[14] P. Pudil, J. Novovicova, and J. Kittler, "Floating search methods in feature selection," Pattern Recognit. Lett., vol. 15, no. 11, pp. 1119-1125, 1994.

[15] P. Somol, P. Pudil, J. Novovicova, and P. Paclik, "Adaptive floating search methods in feature selection," Pattern Recognit. Lett., vol. 20, no. 11-13, pp. 1157-1163, 1999.

[16] C.-I. Chang and Q. Du, "Estimation of number of spectrally distinct signal sources in hyperspectral imagery," IEEE Trans. Geosci. Remote Sens., vol. 46, no. 3, pp. 608-619, Mar. 2004.

[17] J. M. Bioucas-Dias and J. M. P. Nascimento, "Hyperspectral subspace identification," IEEE Trans. Geosci. Remote Sens., vol. 42, no. 8, pp. 2435-2445, Aug. 2008.

[18] A. Plaza and C.-I. Chang, "Impact of initialization on design of endmember extraction algorithm," IEEE Trans. Geosci. Remote Sens., vol. 44, no. 11, pp. 3397-3407, Nov. 2006. 